\title{
BOLD cerebrovascular reactivity as a novel marker for crossed cerebellar diaschisis
}

Sebök, Martina ; van Niftrik, Christiaan H B ; Piccirelli, Marco ; Bozinov, Oliver ; Wegener, Susanne ; Esposito, Giuseppe ; Pangalu, Athina ; Valavanis, Antonios ; Buck, Alfred ; Luft, Andreas R ; Regli, Luca ; Fierstra, Jorn

\begin{abstract}
OBJECTIVE To study blood oxygen level-dependent cerebrovascular reactivity (BOLD-CVR) as a surrogate imaging marker for crossed cerebellar diaschisis (CCD). METHODS Twenty-five participants with symptomatic unilateral cerebrovascular steno-occlusive disease underwent a BOLD-CVR and an acetazolamide challenged (O)-HO-PET study. CCD and cerebellar asymmetry index were determined from PET and compared to BOLD-CVR quantitative values. Neurologic status at admission and outcome after 3 months were determined with NIH Stroke Scale (NIHSS) and modified Rankin Scale (mRS) scores. RESULTS For both the BOLD-CVR and PET examination, a significant cerebellar asymmetry index was found for participants exhibiting CCD (CCD+ vs CCD-: for BOLD-CVR $13.11 \pm 9.46$ vs $1.52 \pm 4.97,<0.001$; and for PET $7.31 \pm 2.75$ vs $1.68 \pm 2.98,<0.001)$. The area under the curve for BOLD-CVR was 0.89 (95\% confidence interval: $0.75-1.0)$ with 0.91 sensitivity and 0.81 specificity to detect CCD. Participants exhibiting CCD were in poorer clinical condition at baseline (CCD+ vs CCD-: NIHSS 7 vs $1,=0.003 ; \mathrm{mRS} 3$ vs $1,=0.001$ ) and after 3 -month follow-up (NIHSS 2 vs $0,=0.02 ; \mathrm{mRS}$ 1 vs $0,=0.04)$. Worse performance on both scores showed an agreement with a larger BOLD-CVR cerebellar asymmetry index. This was not found for PET. CONCLUSIONS BOLD-CVR demonstrates similar sensitivity to detect CCD as compared to (O)-HO-PET in patients with symptomatic unilateral cerebrovascular steno-occlusive disease. Furthermore, participants exhibiting CCD had a poorer baseline neurologic performance and neurologic outcome at 3 months. CLASSIFICATION OF EVIDENCE This study provides Class II evidence that BOLD-CVR identifies CCD in patients with symptomatic unilateral cerebrovascular steno-occlusive disease.
\end{abstract}

DOI: https://doi.org/10.1212/WNL.0000000000006287

Posted at the Zurich Open Repository and Archive, University of Zurich

ZORA URL: https://doi.org/10.5167/uzh-153754

Journal Article

Published Version

Originally published at:

Sebök, Martina; van Niftrik, Christiaan H B; Piccirelli, Marco; Bozinov, Oliver; Wegener, Susanne; Esposito, Giuseppe; Pangalu, Athina; Valavanis, Antonios; Buck, Alfred; Luft, Andreas R; Regli, Luca; Fierstra, Jorn (2018). BOLD cerebrovascular reactivity as a novel marker for crossed cerebellar diaschisis. Neurology, 91(14):e1328-e1337.

DOI: https://doi.org/10.1212/WNL.0000000000006287 


\section{BOLD cerebrovascular reactivity as a novel marker for crossed cerebellar diaschisis}

Martina Sebök, MD, * Christiaan H.B. van Niftrik, MD,* Marco Piccirelli, PhD, Oliver Bozinov, MD, Susanne Wegener, MD, Giuseppe Esposito, MD, PhD, Athina Pangalu, MD, Antonios Valavanis, MD, Alfred Buck, MD, Andreas R. Luft, MD, Luca Regli, MD, and Jorn Fierstra, MD, PhD

Neurology ${ }^{\circledR}$ 2018;00:e1-e10. doi:10.1212/WNL.0000000000006287

\section{Abstract}

\section{Objective}

To study blood oxygen level-dependent cerebrovascular reactivity (BOLD-CVR) as a surrogate imaging marker for crossed cerebellar diaschisis (CCD).

\section{Methods}

Twenty-five participants with symptomatic unilateral cerebrovascular steno-occlusive disease underwent a BOLD-CVR and an acetazolamide challenged $\left({ }^{15} \mathrm{O}\right)-\mathrm{H}_{2} \mathrm{O}-\mathrm{PET}$ study. CCD and cerebellar asymmetry index were determined from PET and compared to BOLD-CVR quantitative values. Neurologic status at admission and outcome after 3 months were determined with NIH Stroke Scale (NIHSS) and modified Rankin Scale (mRS) scores.

\section{Results}

For both the BOLD-CVR and PET examination, a significant cerebellar asymmetry index was found for participants exhibiting CCD (CCD+ vs CCD-: for BOLD-CVR $13.11 \pm 9.46$ vs 1.52 $\pm 4.97, p<0.001$; and for PET $7.31 \pm 2.75$ vs $1.68 \pm 2.98, p<0.001)$. The area under the curve for BOLD-CVR was 0.89 (95\% confidence interval: $0.75-1.0$ ) with 0.91 sensitivity and 0.81 specificity to detect CCD. Participants exhibiting CCD were in poorer clinical condition at baseline (CCD+ vs CCD-: NIHSS 7 vs $1, p=0.003$; $\mathrm{mRS} 3$ vs $1, p=0.001$ ) and after 3 -month follow-up (NIHSS 2 vs $0, p=0.02$; mRS 1 vs $0, p=0.04$ ). Worse performance on both scores showed an agreement with a larger BOLD-CVR cerebellar asymmetry index. This was not found for PET.

\section{Conclusions}

BOLD-CVR demonstrates similar sensitivity to detect CCD as compared to $\left({ }^{15} \mathrm{O}\right)-\mathrm{H}_{2} \mathrm{O}-\mathrm{PET}$ in patients with symptomatic unilateral cerebrovascular steno-occlusive disease. Furthermore, participants exhibiting CCD had a poorer baseline neurologic performance and neurologic outcome at 3 months.

\section{Classification of evidence}

This study provides Class II evidence that BOLD-CVR identifies CCD in patients with symptomatic unilateral cerebrovascular steno-occlusive disease.
Correspondence

Dr. Fierstra

jorn.fierstra@usz.ch

\section{MORE ONLINE}

$\rightarrow$ Class of Evidence

Criteria for rating

therapeutic and diagnostic studies

NPub.org/coe

\footnotetext{
*These authors contributed equally to this work.
}

From the Department of Neurosurgery (M.S., C.H.B.v.N., O.B., G.E., L.R., J.F.), Clinical Neuroscience Center (M.S., C.H.B.v.N., M.P., O.B., S.W., G.E., A.P., A.V., A.R.L., L.R., J.F.), and Departments of Neuroradiology (M.P., A.P., A.V.), Neurology (S.W., A.R.L.), and Nuclear Medicine (A.B.), University Hospital Zurich, University of Zurich, Switzerland.

Go to Neurology.org/N for full disclosures. Funding information and disclosures deemed relevant by the authors, if any, are provided at the end of the article. 


\section{Glossary}

$\mathbf{B O L D}=$ blood oxygen level-dependent $\mathbf{C C D}=$ crossed cerebellar diaschisis; $\mathbf{C I}=$ confidence interval; $\mathbf{C V R}=$ cerebrovascular reactivity; MNI = Montreal Neurological Institute; $\mathbf{m R S}=$ modified Rankin Scale; NIHSS = NIH Stroke Scale.

Crossed cerebellar diaschisis (CCD), known as a functional disconnection of the cerebellar hemisphere contralateral to a supratentorial lesion, is associated with poor clinical outcome but remains solely a radiologic diagnosis. ${ }^{1-3}$ The subsequent reduction in both blood flow and oxygen extraction fraction in the affected cerebellar hemisphere is traditionally detected with $\left({ }^{15} \mathrm{O}\right)-\mathrm{H}_{2} \mathrm{O}-\mathrm{PET}{ }^{2,4-9}$ Contemporary MRI techniques, such as dynamic susceptibility contrast $\mathrm{MRI}^{10-12}$ and arterial spin labeling, ${ }^{13,14}$ however, may be favored because of better clinical accessibility and absence of exogenous radioisotopes.

In this regard, the application of blood oxygen leveldependent (BOLD) fMRI also deserves further investigation. From BOLD in combination with a carbon dioxide challenge, cerebrovascular reactivity (CVR) can be quantitatively mapped for the entire brain, including the cerebellum. ${ }^{15-17}$ BOLD-CVR is defined as the percentage BOLD signal change per $\mathrm{mm} \mathrm{Hg}$ carbon dioxide and is frequently used to measure supratentorial cerebrovascular reserve capacity. ${ }^{18}$ Although BOLD signal changes can be seen in parallel with changes in cerebral blood flow, ${ }^{19}$ the BOLD signal primarily depends on changes in deoxyhemoglobin-not cerebral blood flow changes directly. In participants with $C C D$, the presence of hypometabolism in the affected cerebellar hemisphere results in less oxyhemoglobin being converted to deoxyhemoglobin. This leads to less deoxyhemoglobin outwash after a physiologic cerebral blood flow increase (i.e., preserved cerebral blood flow reactivity in acetazolamide challenged PET imaging) and will subsequently lead to a dampened BOLD signal increase. $^{20,21}$

Although a recent study ${ }^{22}$ has demonstrated good feasibility of BOLD-CVR for detecting CCD, its diagnostic capacity and prognostic value remain to be studied in more detail. Therefore, our aim was to further investigate the role of BOLD-CVR for CCD.

\section{Methods}

\section{Primary research question}

Can BOLD-CVR identify CCD in patients with symptomatic unilateral cerebrovascular steno-occlusive disease?

\section{Classification of evidence}

This study provides Class II evidence that BOLD-CVR identifies CCD in patients with symptomatic unilateral cerebrovascular steno-occlusive disease.

\section{Standard protocol approvals, registrations, and patient consents}

The research ethics board of the canton Zurich, Switzerland (KEK-ZH-Nr. 2012-0427), approved the study, and all participants signed informed consent before participation.

\section{Participant selection}

Twenty-five participants with symptomatic unilateral cerebrovascular steno-occlusive disease at either the subacute or chronic stroke stage were selected from an ongoing prospective BOLD-CVR database between February 2015 and February 2018. Patients older than 18 years with symptomatic unilateral cerebrovascular steno-occlusive disease treated at University Hospital Zurich who gave informed consent were included in this database. Participants were extracted from this database and included in the study if they had undergone acetazolamide challenged $\left({ }^{15} \mathrm{O}\right)-\mathrm{H}_{2} \mathrm{O}-\mathrm{PET}$ and BOLD-CVR imaging within a time frame of 4 weeks. Participants with an interval more than 4 weeks between PET and BOLD-CVR imaging were excluded because of potential disease alteration. All enrolled participants underwent the same PET imaging as a reference standard. Other exclusion criteria were signs of cerebellar infarction or other cerebellar pathology on initial or follow-up imaging, new neurologic symptoms between both scans, surgical revascularization between both scans and inability or refusal to sign informed consent.

\section{Image acquisition and processing}

\section{BOLD-CVR}

MRI data were acquired on a 3-tesla Skyra VD13 (Siemens Healthcare, Erlangen, Germany) with a 32-channel head coil. BOLD fMRI parameters: axial 2-dimensional single-shot echo-planar imaging sequence planned on the anterior commissure-posterior commissure line plus $20^{\circ}$ (on a sagittal image), voxel size $3 \times 3 \times 3 \mathrm{~mm}^{3}$, acquisition matrix $64 \times 64 \times$ 35 ascending interleaved slice acquisition, slice gap $0.3 \mathrm{~mm}$, GRAPPA (generalized autocalibrating partially parallel acquisitions) factor 2 with 32 reference lines, repetition time/ echo time 2,000/30 milliseconds, flip angle $85^{\circ}$, bandwidth $2,368 \mathrm{~Hz} / \mathrm{Px}$, and field of view $192 \times 192 \mathrm{~mm}^{2}$. Two hundred volumes were acquired for the CVR study. A 3-dimensional, T1-weighted, magnetization-prepared rapid-acquisition gradient echo image was also acquired with the same orientation as the fMRI scans for overlay purposes. Acquisition parameters were as follows: voxel size $0.8 \times 0.8 \times 1.0 \mathrm{~mm}^{3}$ with a field of view $230 \times 230 \times 176 \mathrm{~mm}$ and scan matrix of $288 \times 288 \times$ 176 , repetition time/echo time/inversion time 2,200/5.14/900 milliseconds, and flip angle $8^{\circ}$. The carbon dioxide stimulus was given with a computer-controlled gas blender with prospective gas targeting algorithms (RespirAct; Thornhill Research 
Institute, Toronto, Canada). This stimulus allows for precise targeting of arterial partial pressure of oxygen and carbon dioxide. ${ }^{15}$ We controlled the carbon dioxide at the participant's own resting carbon dioxide value. During the CVR study, carbon dioxide was increased approximately $10 \mathrm{~mm} \mathrm{Hg}$ above their resting carbon dioxide value for 80 seconds. Oxygen was maintained at a level of approximately $100 \mathrm{~mm} \mathrm{Hg}$ during the entire protocol.

\section{Data preprocessing and BOLD-CVR calculations}

All the acquired raw BOLD volumes were transferred and analyzed with SPM 12 (Statistical Parameter Mapping Software; Wellcome Department of Imaging Neuroscience, University College of London, UK). The BOLD volumes were timecorrected and orthogonalized in 6 translational and rotational motion estimates to decrease the influence of head motion. The BOLD volumes were then aligned to the T1-weighted image and normalized in Montreal Neurological Institute (MNI) space of the MNI/ICBM AVG 152 template using a 12-parameter affine linear transformation and warping of the images using a nonlinear transformation. Finally, the BOLD volumes were smoothed with a gaussian kernel with $8-\mathrm{mm}$ full width at half maximum.

The CVR calculations were done according to a previously described and validated method. ${ }^{16}$ In short, after temporal shifting for optimal physiologic correlation of the 2 time series, CVR was calculated from the slope of a linear least square fit of the BOLD signal time course to the carbon dioxide time series over the range of the first baseline of 100 seconds, the 80 seconds step portion of the protocol, and the second baseline of 100 seconds on a voxel-by-voxel basis. CVR was defined as the percentage BOLD signal change per $\mathrm{mm} \mathrm{Hg}$ carbon dioxide change. The extra BOLD volumes were acquired to allow for potential temporal shift. ${ }^{16}$

\section{$\left({ }^{15} \mathrm{O}\right)-\mathrm{H}_{2} \mathrm{O}-\mathrm{PET}$ study}

PET data were acquired on a full-ring PET/CT scanner in 3-dimensional mode (PET/CT Discovery STE; GE Healthcare, Waukesha, WI). The images were corrected for attenuation and scatter by using the manufacturer's own algorithms and a corresponding CT $(120 \mathrm{kV} / 80 \mathrm{~mA})$. The total axial field of view was $15.3 \mathrm{~cm}$. Images were reconstructed using a 3-dimensional Fourier rebinning filtered backprojection algorithm giving a $128 \times 128 \times 47$ matrix with $2.34 \times 2.34 \times$ $3.27 \mathrm{~mm}$ voxel spacing. Over a period of approximately 20 seconds, 300 to $800 \mathrm{MBq}$ of $\left({ }^{15} \mathrm{O}\right)-\mathrm{H}_{2} \mathrm{O}$ was administered IV using an automatic injection device. The emission data were acquired as a series of 18, 10-second frames. Acetazolamide was injected approximately 13 minutes before the second PET scan (dose adjusted according to weight) over a period of 2 minutes. Parametric images of cerebral blood flow before and after the acetazolamide challenge were generated using a previously reported method ${ }^{23,24}$ in which a standardized arterial input function and image scaling based on the washout rate $\mathrm{k} 2$ of $\left({ }^{15} \mathrm{O}\right)-\mathrm{H}_{2} \mathrm{O}$ are used to determine cerebral blood flow. This method ${ }^{24}$ utilizes the fact that $\mathrm{k} 2$ is related to the shape and not the scale of the arterial input function and proportional to cerebral blood flow. A total count-rate threshold was set at $50 \%$ of the maximum.

The baseline PET image and acetazolamide PET image were then coregistered to the mean BOLD to allow for normalization of the images. Similar to the BOLD images, normalization of the 2 PET images was done in MNI space and smoothed with a gaussian kernel with $8-\mathrm{mm}$ full width at half maximum. CCD was determined visually based on PET baseline images by an experienced senior staff member of the Department of Nuclear Medicine (A.B.) who was blinded toward the BOLD-CVR findings. For both the PET cerebral blood flow images and the BOLD-CVR image, a cerebellar asymmetry index (ipsilateral [to supratentorial stroke] cerebellar hemisphere - crossed cerebellar hemisphere/ipsilateral hemisphere) was determined using a predefined cerebellar mask to allow for a quantitative analysis of the visually selected CCD-positive and -negative groups. This cerebellar asymmetry index is reported as a percentage.

\section{Assessment of neurologic and functional status}

The NIH Stroke Scale (NIHSS) and modified Rankin Scale (mRS) were used to evaluate the neurologic and functional status at the time of stroke, and at 3-month follow-up.

\section{Statistical analysis}

We performed the statistical analysis using SPSS Statistics 23 (IBM Corp., Armonk, NY). First, normal distribution was evaluated using the Shapiro-Wilk test. Means of normally distributed continuous variables from the CCD-positive and CCD-negative groups were compared by an independent Student 2 -tailed $t$ test, where $p<0.05$ was considered statistically significant. Variables with nonnormal distribution were analyzed using the Mann-Whitney $U$ test. All normally distributed continuous variables are reported as mean \pm SD. Categorical ordinal variables are presented as median (interquartile range) whereas dichotomous variables are shown as frequency (\%). To evaluate cerebral blood flow reactivity in PET imaging as well as the quantitative intrasubject cerebellar hemisphere CVR differences, we used a paired sample $t$ test. For the assessment of detecting CCD, a receiver operating characteristic curve and the area under the curve were calculated, including a confidence interval (CI). A Bland-Altman analysis was used to compare mean differences in cerebellar asymmetry index between BOLD-CVR and PET baseline imaging, and the results were plotted to evaluate the agreement between the 2 techniques and potential bias.

To identify a relationship between the cerebellar asymmetry index, detected by either BOLD-CVR or one of the PET images, and neurologic outcome after 3 months, measured using NIHSS and mRS, we used a Spearman rank-order correlation analysis.

\section{Data availability}

In addition to the detailed methodologic description provided in this report, numeric data of the individual 27 datasets can 
also be made available in anonymized format. Please contact the corresponding author for such a request.

\section{Results}

From the prospective database, 33 participants underwent both $\left({ }^{15} \mathrm{O}\right)-\mathrm{H}_{2} \mathrm{O}$-PET and BOLD-CVR scans resulting in 35 datasets that were considered for inclusion. After evaluation of exclusion criteria, 25 participants were eligible for further analysis. Two participants qualified for inclusion twice. One participant fulfilled the inclusion criteria twice as he experienced 2 ischemic events on separate occasions, while a second participant underwent pre- and postsurgical imaging after extracranial-tointracranial bypass revascularization. Eight patients were excluded because the time period between PET and BOLDCVR scans exceeded 4 weeks and one patient was excluded because of missed follow-up scans. In total, 27 combined BOLD-CVR and PET studies were performed in 25 participants. Mean age was $57 \pm 15$ years and $70 \%$ of the participants were men. The clinical and relevant baseline characteristics of all participants are shown in tables 1 and 2. Of the 27 datasets, 11 (41\%) were classified as CCD positive. None of the 10 participants ( 11 datasets) with CCD positivity showed clinical signs of cerebellar dysfunction. Two participants from the CCDnegative group and one participant from the CCD-positive group underwent thrombolysis previous to the BOLD and PET scans. Exemplary imaging illustrations of one CCD-positive and one CCD-negative participant are shown in figure 1.

Table 1 Participant characteristics

\begin{tabular}{|c|c|c|c|c|c|}
\hline Participant & Age, y & Sex & Diagnosis (TOAST) & $\begin{array}{l}\text { Angiographic } \\
\text { findings }\end{array}$ & $\begin{array}{l}\text { Time from ischemic } \\
\text { event to imaging }\end{array}$ \\
\hline 1 & 78 & $\mathrm{M}$ & Carotid atherosclerosis (TOAST 1) & L ICA occlusion & $79 \mathrm{mo}$ \\
\hline 2 & 33 & $\mathrm{~F}$ & Spontaneous dissection (TOAST 4) & L ICA dissection & $1 \mathrm{wk}$ \\
\hline 3 & 44 & $\mathrm{~F}$ & Moyamoya disease (TOAST 4) & R MCA stenosis & $57 \mathrm{mo}$ \\
\hline 4 & 47 & $\mathrm{M}$ & Carotid atherosclerosis (TOAST 1) & L ICA occlusion & $21 \mathrm{mo}$ \\
\hline 5 & 67 & $\mathrm{~F}$ & Carotid atherosclerosis (TOAST 1) & L ICA stenosis & $1 \mathrm{mo}$ \\
\hline 6 & 81 & $\mathrm{M}$ & Carotid atherosclerosis (TOAST 1) & R ICA stenosis & 1 and $5 \mathrm{mo}^{\mathrm{a}}$ \\
\hline 7 & 65 & $\mathrm{M}$ & Carotid atherosclerosis (TOAST 1) & R ICA occlusion & $3 w k$ \\
\hline 8 & 62 & $\mathrm{~F}$ & Intracranial stenosis (TOAST 1) & L MCA occlusion & $3 w k$ \\
\hline 9 & 63 & $\mathrm{M}$ & Moyamoya-like collateral supply (TOAST 4) & R MCA stenosis & $13 \mathrm{mo}$ \\
\hline 10 & 63 & $\mathrm{M}$ & Intracranial stenosis (TOAST 1) & L MCA stenosis & $2 w k$ \\
\hline 11 & 76 & $\mathrm{M}$ & Carotid atherosclerosis (TOAST 1) & R ICA stenosis & $1 \mathrm{mo}$ \\
\hline 12 & 52 & $\mathrm{M}$ & Carotid atherosclerosis (TOAST 1) & L ICA occlusion & $10 \mathrm{mo}$ \\
\hline 13 & 51 & $\mathrm{~F}$ & Moyamoya disease (TOAST 4) & R MCA stenosis & $36 \mathrm{mo}$ \\
\hline 14 & 59 & M & Carotid atherosclerosis (TOAST 1) & R ICA stenosis & $1 \mathrm{mo}$ \\
\hline 15 & 77 & $\mathrm{~F}$ & Carotid atherosclerosis (TOAST 1) & L ICA stenosis & $1 \mathrm{mo}$ \\
\hline 16 & 50 & $\mathrm{M}$ & Carotid atherosclerosis (TOAST 1) & R ICA stenosis & 1 and $6 \mathrm{mo}^{\mathrm{a}}$ \\
\hline 17 & 50 & $\mathrm{~F}$ & Moyamoya disease (TOAST 4) & L MCA stenosis & $4 \mathrm{mo}$ \\
\hline 18 & 65 & M & Intracranial stenosis (TOAST 1) & R MCA occlusion & $2 \mathrm{mo}$ \\
\hline 19 & 48 & $\mathrm{M}$ & Intracranial stenosis (TOAST 1) & R MCA occlusion & $1 \mathrm{mo}$ \\
\hline 20 & 45 & $\mathrm{M}$ & Intracranial stenosis (TOAST 1) & R MCA stenosis & $3 w k$ \\
\hline 21 & 70 & $\mathrm{M}$ & Carotid atherosclerosis (TOAST 1) & L ICA occlusion & $1 \mathrm{wk}$ \\
\hline 22 & 40 & $\mathrm{~F}$ & Moyamoya disease (TOAST 4) & R MCA stenosis & $21 \mathrm{mo}$ \\
\hline 23 & 46 & $\mathrm{M}$ & Moyamoya disease (TOAST 4) & L MCA stenosis & $1 \mathrm{mo}$ \\
\hline 24 & 52 & $\mathrm{M}$ & Moyamoya disease (TOAST 4) & L MCA stenosis & $1 \mathrm{mo}$ \\
\hline 25 & 69 & $\mathrm{M}$ & Carotid atherosclerosis (TOAST 1) & R ICA occlusion & $1 \mathrm{wk}$ \\
\hline
\end{tabular}

Abbreviations: ICA = internal carotid artery; MCA = middle cerebral artery; TOAST classification = Trial of ORG 10172 in Acute Stroke Treatment. ${ }^{42}$ a Participant underwent the imaging protocol twice. 
Table 2 Relevant clinical and baseline characteristics

\begin{tabular}{|c|c|c|c|c|}
\hline & $\begin{array}{l}\text { Total cohort } \\
(n=27)\end{array}$ & $\begin{array}{l}\text { CCD-positive } \\
\text { group }(n=11)^{a}\end{array}$ & $\begin{array}{l}\text { CCD-negative } \\
\text { group }(n=16)^{a}\end{array}$ & $p$ Value \\
\hline Age, y & $57 \pm 15$ & $56 \pm 10$ & $60 \pm 15$ & 0.43 \\
\hline Sex, male & $19(70)$ & $8(73)$ & $11(69)$ & 0.83 \\
\hline Smoking & $13(48)$ & $6(55)$ & $7(44)$ & 0.60 \\
\hline Hypertension & $18(67)$ & $7(64)$ & $11(69)$ & 0.79 \\
\hline Hypercholesterolemia & $10(37)$ & $5(45)$ & $5(31)$ & 0.47 \\
\hline Obesity & $5(19)$ & $1(9)$ & $4(25)$ & 0.31 \\
\hline Diabetes & $2(7)$ & $1(9)$ & $1(6)$ & 0.79 \\
\hline Mean $\mathrm{CO}_{2}$ baseline, $\mathrm{mm} \mathrm{Hg}$ & $38.1 \pm 2.6$ & $38.2 \pm 2.6$ & $38.2 \pm 2.6$ & 1 \\
\hline Mean $\mathrm{CO}_{2}$ hypercapnia, $\mathrm{mm} \mathrm{Hg}$ & $47.1 \pm 2.5$ & $47.5 \pm 2.2$ & $46.8 \pm 2.7$ & 0.44 \\
\hline Mean $\mathrm{CO}_{2}$ step change, $\mathrm{mm} \mathrm{Hg}$ & $8.8 \pm 1.8$ & $9.3 \pm 1.6$ & $8.5 \pm 1.9$ & 0.29 \\
\hline
\end{tabular}

Abbreviation: $C C D$ = crossed cerebellar diaschisis.

Data represent mean \pm SD or $\mathrm{n}(\%)$.

${ }^{a}$ One patient in each group underwent the protocol 2 times.

\section{BOLD-CVR and PET findings}

The CVR cerebellar asymmetry index was different in CCDpositive participants compared with CCD-negative participants $(13.11 \pm 9.46$ vs $1.52 \pm 4.97, p<0.001)$. Similar to the CVR cerebellar asymmetry index, baseline PET cerebellar asymmetry index was also asymmetrical in the CCD-positive group as compared with the CCD-negative group ( $7.31 \pm 2.75$ vs $1.68 \pm 2.98, p<0.001)$. The PET cerebellar asymmetry index did not differ before and after the acetazolamide challenge (CCD positive: $7.31 \pm 2.75$ vs $6.93 \pm 1.90, p=0.6$; CCD negative: $1.68 \pm 2.98$ vs $0.94 \pm 2.61, p=0.3$ ), indicating preserved cerebral blood flow reactivity. Supratentorial CVR in CCD-positive participants was more impaired in the ipsilateral hemisphere as compared to the ipsilateral hemisphere of CCD-negative participants $(0.08 \pm 0.09$ vs $0.16 \pm 0.07, p=$ $0.01)$. No difference was seen for the contralateral hemisphere. All BOLD-CVR and PET findings are shown in table 3 .

The receiver operating characteristic curve and BlandAltman analysis are presented in figure 2. Here, BOLD-CVR shows an area under the curve of 0.89 (95\% CI: 0.75-1.0). This area under the curve of BOLD-CVR was not different from the PET imaging (area under the curve PET cerebellar asymmetry index: 0.94, 95\% CI: 0.85-1.0). The receiver operating characteristic curve showed that with a cutoff point of $6 \%$ BOLD-CVR cerebellar asymmetry index, CCD could be detected with a sensitivity of 0.91 and a specificity of 0.81 . The cutoff points for PET baseline cerebellar asymmetry index and PET Diamox cerebellar asymmetry index were $4.2 \%$ and $5.0 \%$, respectively. The Bland-Altman analysis showed a proportional bias when combining data of both groups $\left(r^{2}=0.7, p<0.001\right.$; figure $\left.2 \mathrm{~B}\right)$. Considering the data of the CCD-negative participants alone, a good agreement was found without proportional bias $\left(r^{2}=0.30\right.$, $p=0.1$; figure $2 \mathrm{C}$ ).

\section{Clinical status and outcome assessments}

Clinical status and outcome measurements are presented in table 4 and figure 3. Differences between the CCD-positive and CCD-negative groups were found for both clinical scores at the time of stroke (CCD+ vs CCD-: mean [interquartile]: NIHSS 7 [5] vs 1 [2], $p=0.003$; mRS 3 [1] vs 1 [2], $p=$ $0.001)$. This difference persisted at 3-month follow-up (NIHSS $2[5]$ vs $0[0], p=0.02$; mRS 1 [2] vs $0[1], p=$ 0.04). Of note, both groups showed an improvement of both scores at 3-month follow-up.

The relationship between the cerebellar asymmetry index, detected by either BOLD-CVR or one of the PET images, and neurologic outcome was calculated using the NIHSS and mRS scores at 3 months. An increasing BOLD-CVR cerebellar asymmetry index showed good agreement with worse performance on both the NIHSS $(r=0.52, p=0.02)$ and $\mathrm{mRS}$ $(r=0.56, p=0.01)$. Contrarily, the PET cerebellar asymmetry index measurements did not (baseline PET: NIHSS: $r=0.31$, $p=0.19$; mRS: $r=0.32, p=0.19$; acetazolamide PET: NIHSS: $r=0.28, p=0.25$, mRS: $r=0.15, p=0.53)$.

\section{Discussion}

The main finding of our study is that, similar to baseline and acetazolamide challenged $\left({ }^{15} \mathrm{O}\right)-\mathrm{H}_{2} \mathrm{O}-\mathrm{PET}$, BOLD-CVR can detect the presence of $\mathrm{CCD}$ with high specificity and sensitivity. Furthermore, the presence of CCD corresponds to a poorer neurologic status at baseline and at 3-month followup, a finding in concordance with previous studies. ${ }^{1,2,25}$ It is of 

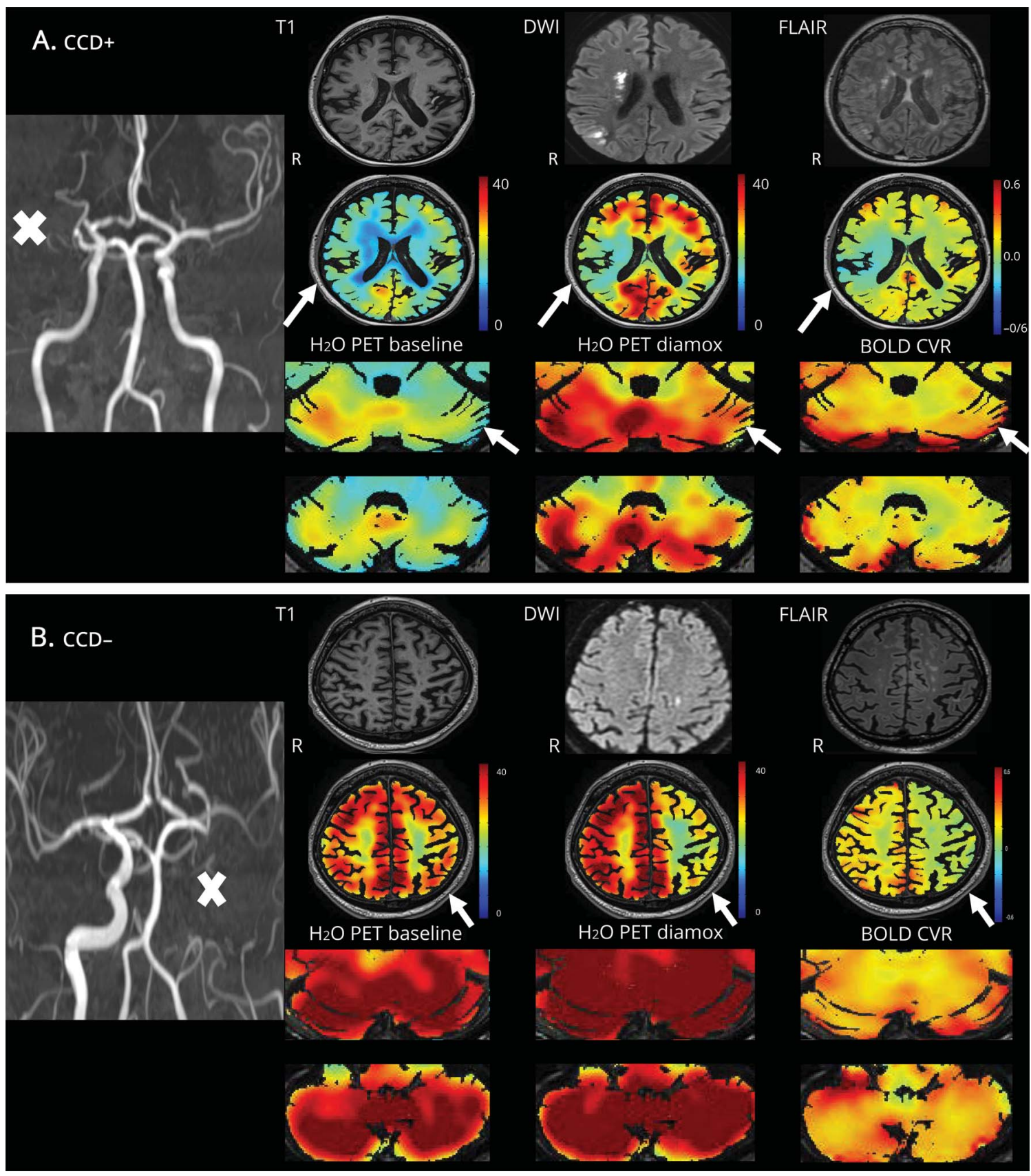

(A) A 48-year-old man with ischemia in the right middle cerebral artery territory due to a middle cerebral artery occlusion (white $\mathrm{x}$ ). He presented with left facial palsy, left-sided hemiparesis, and dysphasia (NIHSS 9/42, mRS 4). On PET and BOLD-CVR imaging, clear signs of supratentorial hemodynamic impairment can be seen in right middle cerebral artery territory (white arrow). In his cerebellum, cerebellar asymmetry can be appreciated contralateral to his supratentorial lesion with good spatial agreement between both imaging modalities, i.e., crossed cerebellar diaschisis (white arrow on bottom images). After 3 months, he showed an improvement of his left-sided hemiparesis, facial palsy, and speech (NIHSS 5/42, mRS 2). (B) A 77-year-old man with ischemia in the left paracentral lobule due to a left internal carotid artery occlusion (white $\mathrm{x}$ ). He presented with a mild sensorimotor paresis of his right leg (NIHSS 2/42, mRS 3), which resolved quickly. The participant did not experience any neurologic symptoms after 3 months (NIHSS 0/42, mRS 0). For both PET imaging as well as BOLD-CVR, supratentorial hemodynamic impairment can be appreciated on the left side (white arrow), whereas no crossed cerebellar diaschisis is observed. BOLD = blood oxygen level-dependent; $C C D$ = crossed cerebellar diaschisis; $C V R=$ cerebrovascular reactivity; DWI = diffusion-weighted imaging; FLAIR = fluidattenuated inversion recovery; mRS = modified Rankin Scale; NIHSS = NIH Stroke Scale.

interest that the cerebellar asymmetry index derived from BOLD-CVR showed a strong positive correlation to NIHSS and $\mathrm{mRS}$ stroke severity scores at 3 months.

Others have already reported the feasibility of noninvasive BOLD-CVR as a novel method to study CCD. A recent feasibility study ${ }^{22}$ demonstrated that participants with CCD identified with arterial spin labeling (i.e., cerebellar hemispheric cerebral blood flow asymmetry) also had significantly reduced cerebellar hemispheric BOLD-CVR as compared to control participants without cerebral blood flow asymmetry. BOLD asymmetry was also studied by using a hyperoxic gas 
Table 3 BOLD-CVR and PET findings

\begin{tabular}{|c|c|c|c|c|}
\hline Functional measurement & $\begin{array}{l}\text { Total cohort } \\
(n=27)\end{array}$ & $\begin{array}{l}\text { CCD-positive } \\
\text { group }(n=11)^{a}\end{array}$ & $\begin{array}{l}\text { CCD-negative } \\
\text { group }(n=16)^{a}\end{array}$ & $p$ Value \\
\hline Mean CVR whole brain & $0.15 \pm 0.06$ & $0.13 \pm 0.07$ & $0.17 \pm 0.05$ & $0.04^{\mathrm{b}}$ \\
\hline Mean CVR affected supratentorial hemisphere & $0.13 \pm 0.08$ & $0.08 \pm 0.09$ & $0.16 \pm 0.05$ & $0.01^{\mathrm{b}}$ \\
\hline Mean CVR unaffected supratentorial hemisphere & $0.18 \pm 0.06$ & $0.16 \pm 0.07$ & $0.19 \pm 0.06$ & 0.29 \\
\hline CVR CAI (\%) & $6.24 \pm 9.07$ & $13.11 \pm 9.46$ & $1.52 \pm 4.97$ & $<0.001^{\mathrm{b}}$ \\
\hline PET CAI (\%) baseline & $4.0 \pm 4.0$ & $7.31 \pm 2.75$ & $1.68 \pm 2.98$ & $<0.001^{\mathrm{b}}$ \\
\hline PET CAI (\%) acetazolamide & $3.4 \pm 3.8$ & $6.93 \pm 1.90$ & $0.94 \pm 2.61$ & $<0.001^{\mathrm{b}}$ \\
\hline CVR cerebellar affected (ipsilateral) & $0.2 \pm 0.06$ & $0.20 \pm 0.06$ & $0.21 \pm 0.05$ & 0.47 \\
\hline CVR cerebellar unaffected (crossed) & $0.19 \pm 0.06$ & $0.17 \pm 0.06$ & $0.21 \pm 0.05$ & 0.12 \\
\hline
\end{tabular}

Abbreviations: $\mathrm{BOLD}=$ blood oxygen level-dependent; $\mathrm{CAI}=$ cerebellar asymmetry index; $\mathrm{CCD}=$ crossed cerebellar diaschisis; $\mathrm{CVR}=$ cerebrovascular reactivity, defined as percentage BOLD signal change per $\mathrm{mm} \mathrm{Hg} \mathrm{CO}{ }_{2}$.

Data represent mean \pm SD.

${ }^{a}$ One patient in each group underwent the protocol 2 times.

b Significant.

challenge to show significant asymmetric cerebellar hemispheric BOLD signal changes in a small cohort of participants with acute large-volume middle cerebral artery strokes. ${ }^{26}$ Using a novel carbon dioxide application technique ${ }^{27}$ and improved BOLD-CVR analysis methods, ${ }^{16}$ we were able to generate high-resolution quantitative cerebellar BOLD-CVR maps. With a cutoff point of BOLD-CVR cerebellar asymmetry index of $6, \mathrm{CCD}$ was detected with a sensitivity of 0.91 and a specificity of 0.81 .

\section{Significance of CCD detection}

Our study found $40.7 \%$ of participants with CCD, which is similar to the incidence reported by others. ${ }^{7,28-30}$ Our data confirm that CCD is related to worse neurologic outcome

Figure 2 Agreement between PET and BOLD-CVR
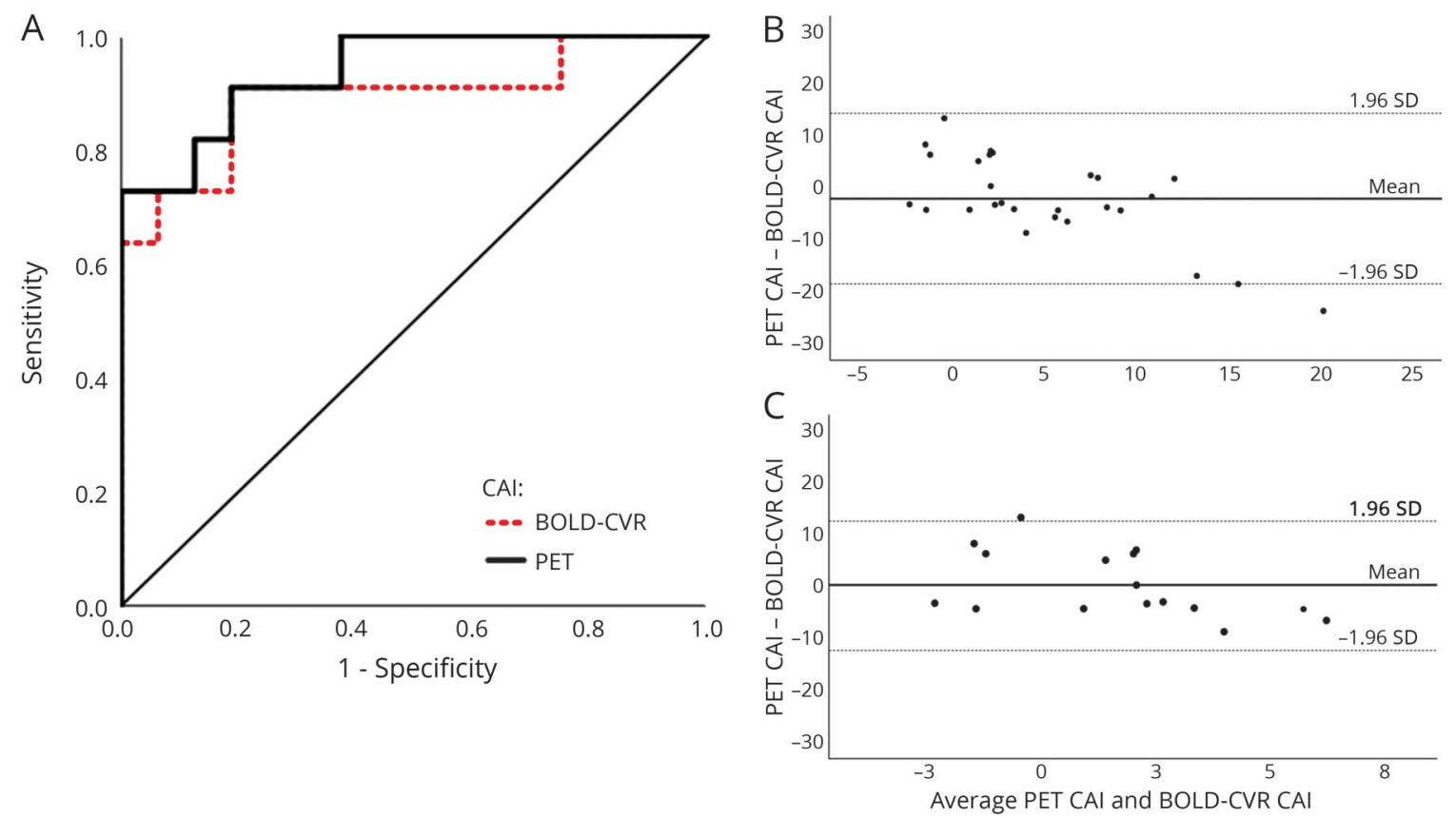

Receiver operating characteristic curve (A) of BOLD-CVR and PET for the detection of crossed cerebellar diaschisis. Bland-Altman plot of the whole cohort (B) and for the crossed cerebellar diaschisis-negative cohort alone (C). Note that a proportional bias can be seen when considering data of both groups. For the crossed cerebellar diaschisis-negative cohort alone (C), a good agreement is found. BOLD = blood oxygen level-dependent; CAI = cerebellar asymmetry index; CVR = cerebrovascular reactivity. 
Table 4 Measurements of functional and neurologic status

\begin{tabular}{|c|c|c|c|c|}
\hline Outcome measurements & $\begin{array}{l}\text { Total cohort } \\
(n=27)\end{array}$ & $\begin{array}{l}\text { CCD-positive } \\
\text { group }(n=11)^{a}\end{array}$ & $\begin{array}{l}\text { CCD-negative } \\
\text { group }(n=16)^{a}\end{array}$ & $p$ Value \\
\hline NHISS during stroke & $2(6)$ & $7(5)$ & $1(2)$ & $0.003^{b}$ \\
\hline mRS during stroke & $2(2)$ & $3(1)$ & $1(2)$ & $0.001^{\mathrm{b}}$ \\
\hline NHISS 3 mo & $0(2)$ & $2(5)$ & $0(0)$ & $0.02^{\mathrm{b}}$ \\
\hline mRS 3 mo & $0(1)$ & $1(2)$ & $0(1)$ & $0.04^{\mathrm{b}}$ \\
\hline
\end{tabular}

Abbreviations: $C C D=$ crossed cerebellar diaschisis; $m R S$ = modified Rankin Scale; NIHSS = NIH Stroke Scale.

All values are median (interquartile).

a One patient in each group underwent the protocol 2 times.

b Significant.

after stroke, as compared to previous studies. ${ }^{1,2,4,25}$ On the contrary, others ${ }^{29,31}$ have shown that the presence of CCD in the acute phase has no effect on functional outcome. It is important to mention that correction of hypoperfusion after acute-stage stroke could shift some participants from CCD positive to CCD negative. ${ }^{2}$ Here, with a PET imaging study, the presence of CCD was assessed at 4 different time points (before thrombolysis, 3 hours, 24 hours, and 14 days later). ${ }^{2}$ Here, participants who recovered from CCD tended to have a more favorable outcome.

\section{CCD data interpretation}

CCD may be caused by an interruption of corticopontocerebellar fiber tracts projecting via pontine nuclei to the contralateral cerebellar cortex and cerebellar nuclei thereby leading to a reduced activation of the cortical Purkinje cells. $^{32,33}$ Conversely, alterations of the reciprocal cerebellothalamocortical tracts may also result in impaired feedback to the cerebrum, resulting in less input from the cerebrum.

The net result is that CCD manifests itself primarily through decreased cerebellar blood flow, oxygen extraction fraction, and cerebral blood volume. ${ }^{22,29}$ In the affected cerebellar hemisphere, the decreased cerebral blood volume and blood flow may occur due to a physiologic vasoconstrictive response to decreased metabolic needs without loss of vasodilatory capacity. Hence, the cerebral blood flow reactivity (i.e., cerebral blood flow change in response to a vasoactive stimulus) remains functional. ${ }^{34-36}$ Our data also showed no difference in PET cerebellar asymmetry index before and after the acetazolamide challenge, thereby confirming a preserved cerebral blood flow reactivity. With a preserved cerebral blood flow reactivity, the reduced cerebellar BOLD-CVR response in participants who are $\mathrm{CCD}$ positive may therefore be explained by the presence of hypometabolism. As a consequence, there is, on average, less conversion of oxyhemoglobin into deoxyhemoglobin. Since the BOLD signal primarily depends on the relative change in deoxyhemoglobin (i.e., oxy-/deoxyhemoglobin ratio) before and after a vasoactive stimulus, BOLD-CVR in the crossed cerebellar hemisphere will be dampened, despite intact cerebral blood flow reactivity. Of note, the different methodology between BOLD-CVR and PET imaging may explain the proportional bias. In participants with CCD, the decrease in cerebellar cerebral blood flow seems not to be in similar proportions as the relative change in metabolism.

\section{Further CCD considerations}

Based on our data and further review of the available literature, we believe that a subdivision of CCD can be proposed. $\mathrm{CCD}$, in some cases, has the potential to regenerate and recover. ${ }^{2,37,38}$ Why CCD resolves in some cases but persists in others is still an open question. Therefore, the presence of an overall more severely impaired supratentorial CVR in our CCD cohort is a potentially very interesting finding. Since not only larger infarctions, but on average overall larger supratentorial hemodynamic impairment is seen in participants with $\mathrm{CCD},{ }^{2,29}$ we postulate that CCD not only occurs in case of direct damage to the corticopontine cerebellar fiber tracts, but that severe acute or chronic supratentorial hemodynamic impairment may also induce CCD. It is known that hemodynamic impairment, in absence of acute stroke, can alter brain structure and function ${ }^{39,40}$ - for instance, the theory of a "hibernating brain concept" in patients with chronic stroke. ${ }^{40}$ This concept postulates the decrease of cortical excitability and increase in resting motor threshold, in case of a misery cerebral perfusion (impaired or negative CVR). Of note, after revascularization, these participants showed an increase in cortical excitability and a decrease in motor resting threshold with alleviation of misery perfusion. ${ }^{40}$ This would explain why recovery of CCD is seen in participants with small infarcts after reperfusion with thrombolysis ${ }^{2}$ and CCD is even found in stenoocclusive disease participants with misery perfusion but without radiologically defined stroke lesions. ${ }^{41}$

From our point of view, we believe a pathophysiologic distinction needs to be made between lesion-induced and hemodynamic-induced CCD, especially as the current definition of CCD still requires the presence of a supratentorial lesion. We will further study this hypothesis in a larger CCD cohort.

\section{Limitations}

Our data must be interpreted in the context of the study design. First, the study cohort was small (i.e., 27 datasets 
Figure 3 NIHSS and mRS scores of CCD-positive and -negative participants

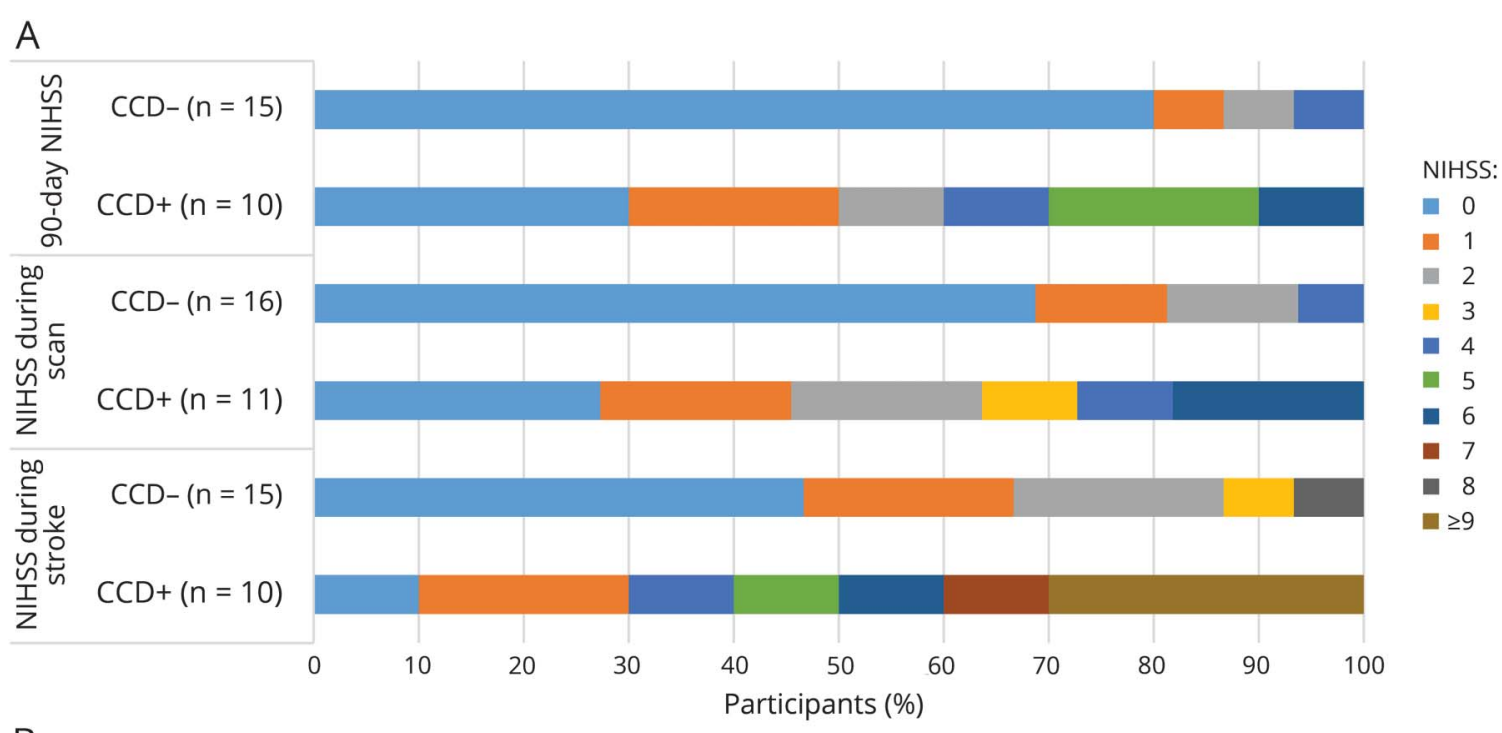

B

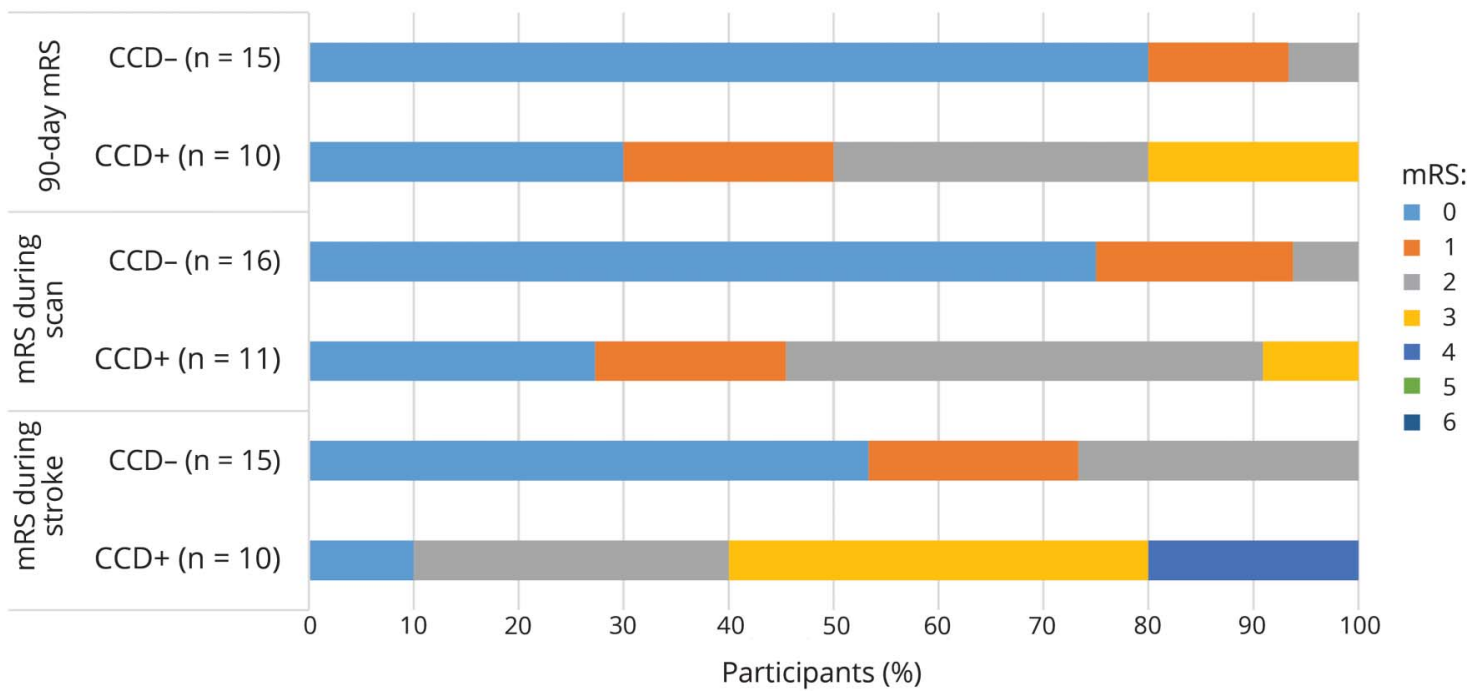

NIHSS scores (A) and mRS scores (B) of CCD-positive and -negative participants. $C C D=$ crossed cerebellar diaschisis; $m$ RS = modified Rankin Scale; NIHSS = NIH Stroke Scale.

from 25 participants), but in concordance with other CCD studies. ${ }^{1,2,8}$ Although our study consisted of a uniform participant population (i.e., those with unilateral steno-occlusive disease), the BOLD-CVR study was performed at either the subacute or chronic stroke stage with a wide range from 1 week to 79 months. Moreover, we cannot comment on acute stroke findings in this participant group. Similarly, as seen in another recent BOLDCVR study, ${ }^{22}$ the cerebral blood flow asymmetry values used to define CCD were based on a region covering a large portion of the cerebellum. Our findings show that hypoperfusion and decreased BOLD signal are not present for the whole cerebellar hemisphere (as can be seen in figure 1A). A more detailed analysis of cerebellar anatomical and functional areas may therefore be considered in future work.

\section{Conclusions}

The main finding of our study is that BOLD-CVR demonstrates similar sensitivity to detect $\mathrm{CCD}$ as compared to $\left({ }^{15} \mathrm{O}\right)-\mathrm{H}_{2} \mathrm{O}$-PET in patients with symptomatic unilateral cerebrovascular steno-occlusive disease. Furthermore, CCD-positive participants had a poorer baseline neurologic performance and neurologic outcome at 3 months. Of interest, a larger BOLD-CVR cerebellar asymmetry index showed good agreement to worse performance on the NIHSS and mRS scales at 3 months. This agreement was not found for the PET cerebellar asymmetry index.

\section{Author contributions}

Dr. Sebök: study concept and design, acquisition of data, analysis and interpretation, constructed the manuscript. 
Dr. van Niftrik: patient selection, study concept and design, acquisition of data, analysis and interpretation, constructed the manuscript. Dr. Piccirelli: acquisition of data, analysis and interpretation, critical revision of the manuscript for important intellectual content. Dr. Bozinov: patient selection, critical revision of the manuscript for important intellectual content, study supervision. Prof. Wegener: critical revision of the manuscript for important intellectual content. Dr. Esposito: critical revision of the manuscript for important intellectual content. Dr. Pangalu: analysis and interpretation, critical revision of the manuscript for important intellectual content. Prof. Valavanis: critical revision of the manuscript for important intellectual content. Prof. Buck: acquisition of data, analysis and interpretation, critical revision of the manuscript for important intellectual content. Prof. Luft: critical revision of the manuscript for important intellectual content. Prof. Regli: patient selection, critical revision of the manuscript for important intellectual content, study supervision. Dr. Fierstra: patient selection, study concept and design, acquisition of data, analysis and interpretation, study supervision.

\section{Study funding}

This work was funded by 2 personal grants obtained by Dr. Jorn Fierstra (Zurich University Forschungskredit; Postdoc 2016, FK-16-040 \& Swiss Cancer League, KFS-3975-082016-R).

\section{Disclosure}

The authors report no disclosures relevant to the manuscript. Go to Neurology.org/N for full disclosures.

Received March 1, 2018. Accepted in final form July 2, 2018.

\section{References}

1. Takasawa M, Watanabe M, Yamamoto S, et al. Prognostic value of subacute crossed cerebellar diaschisis: single-photon emission CT study in patients with middle cerebral artery territory infarct. AJNR Am J Neuroradiol 2002;23:189-193.

2. Sobesky J, Thiel A, Ghaemi M, et al. Crossed cerebellar diaschisis in acute human stroke: a PET study of serial changes and response to supratentorial reperfusion. J Cereb Blood Flow Metab 2005;25:1685-1691.

3. Pantano P, Baron JC, Samson Y, Bousser MG, Derouesne C, Comar D. Crossed cerebellar diaschisis: further studies. Brain 1986;109(pt 4):677-694.

4. De Reuck J, Decoo D, Lemahieu I, Strijckmans K, Goethals P, Van Maele G. Crossed cerebellar diaschisis after middle cerebral artery infarction. Clin Neurol Neurosurg 1997;99:11-16.

5. Kim SE, Choi CW, Yoon BW, et al. Crossed-cerebellar diaschisis in cerebral infarction: technetium-99m-HMPAO SPECT and MRI. J Nucl Med 1997;38:14-19.

6. Kamouchi M, Fujishima M, Saku Y, Ibayashi S, Iida M. Crossed cerebellar hypoperfusion in hyperacute ischemic stroke. J Neurol Sci 2004;225:65-69.

7. Komaba Y, Mishina M, Utsumi K, Katayama Y, Kobayashi S, Mori O. Crossed cerebellar diaschisis in patients with cortical infarction: logistic regression analysis to control for confounding effects. Stroke 2004;35:472-476.

8. Liu Y, Karonen JO, Nuutinen J, Vanninen E, Kuikka JT, Vanninen RL. Crossed cerebellar diaschisis in acute ischemic stroke: a study with serial SPECT and MRI. J Cereb Blood Flow Metab 2007;27:1724-1732.

9. Baron JC, Bousser MG, Comar D, Castaigne P. "Crossed cerebellar diaschisis" in human supratentorial brain infarction. Trans Am Neurol Assoc 1981;105:459-461.

10. Yamada H, Koshimoto Y, Sadato N, et al. Crossed cerebellar diaschisis: assessment with dynamic susceptibility contrast MR imaging. Radiology 1999;210:558-562.

11. Lin DD, Kleinman JT, Wityk RJ, et al. Crossed cerebellar diaschisis in acute stroke detected by dynamic susceptibility contrast MR perfusion imaging. AJNR Am J Neuroradiol 2009;30:710-715.

12. Madai VI, Altaner A, Stengl KL, et al. Crossed cerebellar diaschisis after stroke: can perfusion-weighted MRI show functional inactivation? J Cereb Blood Flow Metab 2011;31:1493-1500.
13. Chalela JA, Alsop DC, Gonzalez-Atavales JB, Maldjian JA, Kasner SE, Detre JA. Magnetic resonance perfusion imaging in acute ischemic stroke using continuous arterial spin labeling. Stroke 2000;31:680-687.

14. Kang KM, Sohn $\mathrm{CH}$, Choi SH, et al. Detection of crossed cerebellar diaschisis in hyperacute ischemic stroke using arterial spin-labeled MR imaging. PLoS One 2017; 12:e0173971.

15. Slessarev M, Han J, Mardimae A, et al. Prospective targeting and control of end-tidal $\mathrm{CO} 2$ and $\mathrm{O} 2$ concentrations. J Physiol 2007;581:1207-1219.

16. van Niftrik $\mathrm{CHB}$, Piccirelli $\mathrm{M}$, Bozinov $\mathrm{O}$, et al. Iterative analysis of cerebrovascular reactivity dynamic response by temporal decomposition. Brain Behav 2017;7:e00705.

17. Fierstra J, Sobczyk O, Battisti-Charbonney A, et al. Measuring cerebrovascular reactivity: what stimulus to use? J Physiol 2013;591:5809-5821.

18. Mutch WA, Mandell DM, Fisher JA, et al. Approaches to brain stress testing: BOLD magnetic resonance imaging with computer-controlled delivery of carbon dioxide. PLoS One 2012;7:e47443.

19. Fierstra J, van Niftrik C, Warnock G, et al. Staging hemodynamic failure with blood oxygen-level-dependent functional magnetic resonance imaging cerebrovascular reactivity. Stroke 2018;49:621-629.

20. Buxton RB. The physics of functional magnetic resonance imaging (fMRI). Rep Prog Phys 2013;76:096601.

21. Ogawa S, Lee TM, Kay AR, Tank DW. Brain magnetic resonance imaging with contrast dependent on blood oxygenation. Proc Natl Acad Sci USA 1990;87: 9868-9872.

22. Strother MK, Buckingham C, Faraco CC, et al. Crossed cerebellar diaschisis after stroke identified noninvasively with cerebral blood flow-weighted arterial spin labeling MRI. Eur J Radiol 2016;85:136-142.

23. Alpert NM, Eriksson L, Chang JY, et al. Strategy for the measurement of regional cerebral blood flow using short-lived tracers and emission tomography. J Cereb Blood Flow Metab 1984;4:28-34.

24. Treyer V, Jobin M, Burger C, Teneggi V, Buck A. Quantitative cerebral H2(15)O perfusion PET without arterial blood sampling, a method based on washout rate. Eur J Nucl Med Mol Imaging 2003;30:572-580.

25. Serrati C, Marchal G, Rioux P, et al. Contralateral cerebellar hypometabolism a predictor for stroke outcome? J Neurol Neurosurg Psychiatry 1994;57:174-179.

26. Dani KA, Santosh C, Brennan D, Hadley DM, Muir KW. Crossed cerebellar diaschisis: insights into oxygen challenge MRI. J Cereb Blood Flow Metab 2012;32: 2114-2117.

27. van Niftrik $\mathrm{CH}$, Piccirelli $\mathrm{M}$, Bozinov $\mathrm{O}$, et al. Fine tuning breath-hold-based cerebrovascular reactivity analysis models. Brain Behav 2016;6:e00426.

28. Sommer WH, Bollwein C, Thierfelder KM, et al. Crossed cerebellar diaschisis in patients with acute middle cerebral artery infarction: occurrence and perfusion characteristics. J Cereb Blood Flow Metab 2016;36:743-754.

29. Kunz WG, Sommer WH, Hohne C, et al. Crossed cerebellar diaschisis in acute ischemic stroke: impact on morphologic and functional outcome. J Cereb Blood Flow Metab 2017;27:3615-3624.

30. Nocun A, Wojczal J, Szczepanska-Szerej H, Wilczynski M, Chrapko B. Quantitative evaluation of crossed cerebellar diaschisis, using voxel-based analysis of Tc- $99 \mathrm{~m} \mathrm{ECD}$ brain SPECT. Nucl Med Rev Cent East Eur 2013;16:31-34.

31. Infeld B, Davis SM, Lichtenstein M, Mitchell PJ, Hopper JL. Crossed cerebellar diaschisis and brain recovery after stroke. Stroke 1995;26:90-95.

32. Cicirata F, Zappala A, Serapide MF, Parenti R, Panto MR, Paz C. Different pontine projections to the two sides of the cerebellum. Brain Res Brain Res Rev 2005;49: 280-294.

33. Kultas-Ilinsky K, Ilinsky IA, Verney C. Glutamic acid decarboxylase isoform $65 \mathrm{im}$ munoreactivity in the motor thalamus of humans and monkeys: gamma-aminobutyric acidergic connections and nuclear delineations. J Comp Neurol 2011;519:2811-2837.

34. Bogsrud TV, Rootwelt K, Russell D, Nyberg-Hansen R. Acetazolamide effect on cerebellar blood flow in crossed cerebral-cerebellar diaschisis. Stroke 1990;21:52-55

35. Ishii K, Kanno I, Uemura K, et al. Comparison of carbon dioxide responsiveness of cerebellar blood flow between affected and unaffected sides with crossed cerebellar diaschisis. Stroke 1994;25:826-830.

36. Ito H, Kanno I, Shimosegawa E, Tamura H, Okane K, Hatazawa J. Hemodynamic changes during neural deactivation in human brain: a positron emission tomography study of crossed cerebellar diaschisis. Ann Nucl Med 2002;16:249-254.

37. Di Piero V, Chollet F, Dolan RJ, Thomas DJ, Frackowiak R. The functional nature of cerebellar diaschisis. Stroke 1990;21:1365-1369.

38. Pantano P, Lenzi GL, Guidetti B, et al. Crossed cerebellar diaschisis in patients with cerebral ischemia assessed by SPECT and 123I-HIPDM. Eur Neurol 1987;27:142-148

39. Fierstra J, Maclean DB, Fisher JA, et al. Surgical revascularization reverses cerebral cortical thinning in patients with severe cerebrovascular steno-occlusive disease. Stroke 2011;42:1631-1637.

40. Jussen D, Zdunczyk A, Schmidt S, et al. Motor plasticity after extra-intracranial bypass surgery in occlusive cerebrovascular disease. Neurology 2016;87:27-35.

41. Matsumoto Y, Oikawa K, Nomura JI, et al. Optimal brain 99mtc-ethyl cysteinate dimer SPECT imaging and analysis to detect misery perfusion on 150 PET imaging in patients with chronic occlusive disease of unilateral major cerebral artery. Clin Nucl Med 2017;42:499-505.

42. Chung JW, Park SH, Kim N, et al. Trial of ORG 10172 in Acute Stroke Treatment (TOAST) classification and vascular territory of ischemic stroke lesions diagnosed by diffusion-weighted imaging. J Am Heart Assoc 2014;3:e001119. 


\section{Neurology}

BOLD cerebrovascular reactivity as a novel marker for crossed cerebellar diaschisis Martina Sebök, Christiaan H.B. van Niftrik, Marco Piccirelli, et al.

Neurology published online September 5, 2018

DOI 10.1212/WNL.0000000000006287

This information is current as of September 5, 2018

$\begin{array}{ll}\text { Updated Information \& } & \text { including high resolution figures, can be found at: } \\ \text { http://n.neurology.org/content/early/2018/09/05/WNL.0000000000006 } \\ \text { 287.full } \\ \text { This article, along with others on similar topics, appears in the } \\ \text { following collection(s): } \\ \text { All Cerebrovascular disease/Stroke } \\ \text { http://n.neurology.org/cgi/collection/all_cerebrovascular_disease_strok } \\ \text { e } \\ \text { fMRI } \\ \text { http://n.neurology.org/cgi/collection/fmri } \\ \text { PET } \\ \text { http://n.neurology.org/cgi/collection/pet } \\ \text { Prognosis } \\ \text { http://n.neurology.org/cgi/collection/prognosis } \\ \\ \text { Information about reproducing this article in parts (figures,tables) or in } \\ \text { its entirety can be found online at: } \\ \text { http://www.neurology.org/about/about_the_journal\#permissions } \\ \text { Information about ordering reprints can be found online: } \\ \text { Permissions \& Licensing } & \text { http://n.neurology.org/subscribers/advertise }\end{array}$

Neurology ${ }^{\circledR}$ is the official journal of the American Academy of Neurology. Published continuously since 1951, it is now a weekly with 48 issues per year. Copyright (O 2018 American Academy of Neurology. All rights reserved. Print ISSN: 0028-3878. Online ISSN: 1526-632X.

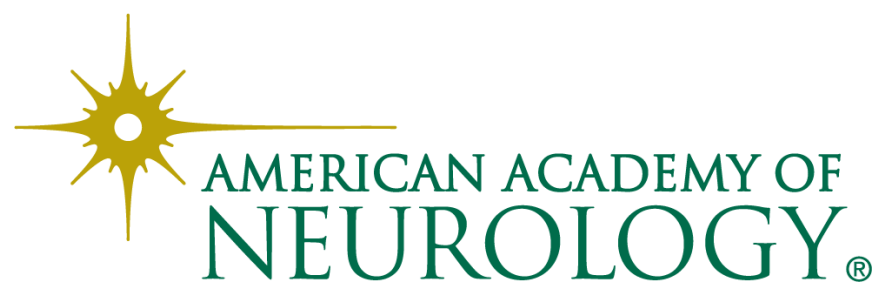

